\title{
Finasteride and Erectile Dysfunction in Patients with Benign Prostatic Hyperplasia or Male Androgenetic Alopecia
}

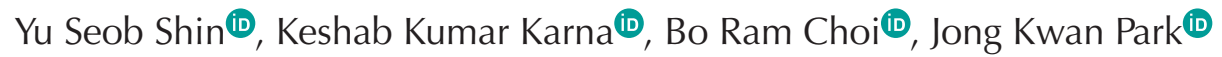 \\ Department of Urology, Chonbuk National University Medical School and Research Institute of Clinical Medicine of Chonbuk National \\ University-Biomedical Research Institute and Clinical Trial Center of Medical Device of Chonbuk National University Hospital, Jeonju, \\ Korea
}

\begin{abstract}
Finasteride is primarily used to treat benign prostatic hyperplasia $(\mathrm{BPH})$ and male androgenetic alopecia (MAA). Five-alpha reductase inhibitors ( $5 \alpha$-RIs) could induce male sexual dysfunction due to their effects on testosterone and dihydrotestosterone. There is evidence suggesting that $5 \alpha$-RIs may independently increase the risk of erectile dysfunction (ED). However, many investigators believe that side effects of $5 \alpha$-RIs will disappear with continuous treatment. Considerable controversy exists regarding the severity and persistence of side effects of finasteride on ED. The aim of this review was to summarize current research studies on finasteride associated with ED. The search strategy used each term of finasteride and ED against PubMed database to identify related studies. ED data reported from available trials for finasteride were summarized and reviewed. Although there is not enough evidence to prove the relationship between finasteride and ED, most studies in this review found that finasteride for $\mathrm{BPH}$ was correlated with $\mathrm{ED}$. However, most studies included in this review revealed that finasteride for MAA was not correlated with ED. On the other hand, some studies reported side effects of finasteride associated with sexual dysfunction, including ED, male infertility, ejaculation problem, and loss of libido, even in MAA patients. Well-designed randomized controlled trials are needed to further determine the mechanism and effects of finasteride on ED. However, physicians should discuss with their patients possible long-term effects of finasteride on sexual function, although we do not have evidence showing that adverse events of sexual dysfunction are absolutely associated with $5 \alpha$-RIs.
\end{abstract}

Keywords: Alopecia; Erectile dysfunction; Finasteride; Prostatic hyperplasia

This is an Open Access article distributed under the terms of the Creative Commons Attribution Non-Commercial License (http://creativecommons.org/licenses/by-nc/4.0) which permits unrestricted non-commercial use, distribution, and reproduction in any medium, provided the original work is properly cited.

\section{INTRODUCTION}

Benign prostatic hyperplasia $(\mathrm{BPH})$ is the main reason for lower urinary tract symptoms (LUTS) due to urethral blockade [1]. There are two kinds of treat- ments for patients with BPH: medical treatment and surgical treatment [2]. Medical treatment using alphablockers or/and 5-alpha reductase inhibitors (5 $\alpha$-RIs) is the first-line treatment for BPH [3]. Finasteride is a type II and type III $5 \alpha-R I s$ [4]. Finasteride $5 \mathrm{mg}$ is

Received: Apr 17, 2018 Revised: May 8, 2018 Accepted: May 23, 2018 Published online August 10, 2018

Correspondence to: Jong Kwan Park iD https://orcid.org/0000-0001-9682-2081

Department of Urology, Chonbuk National University and Research Institute of Clinical Medicine of Chonbuk National University-Biomedical Research Institute and Clinical Trial Center of Medical Device of Chonbuk National University Hospital, 20 Geonji-ro, Deokjin-gu, Jeonju 54907, Korea.

Tel: +82-63-250-1510, Fax: +82-63-250-1564, E-mail: rain@chonbuk.ac.kr 
used to treat symptomatic BPH to shrink the prostate and reduce the risk of urinary retention caused by restricted urine flow when prostate becomes enlarged. Improving symptoms of $\mathrm{BPH}$ with finasteride $5 \mathrm{mg}$ also reduces the need for surgery.

Finasteride is also approved for treating male androgenetic alopecia (MAA) at low dose. Dihydrotestosterone (DHT) is thought to play a significant role in inducing MAA. It is formed from the conversion of circulating testosterone to DHT by 5 -alpha reductase

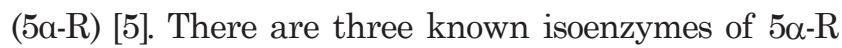
receptors. Type I and type II $5 \alpha-R$ play an important role in the treatment of MAA [6]. Type I $5 \alpha-\mathrm{R}$ is predominantly located in the skin, including sebaceous glands and hair follicles. Type II $5 \alpha-\mathrm{R}$ is the major contributor to DHT pool. It is located in the inner root sheath of hair follicles in the scalp, beard, and chest as well as genitals and prostate gland. Type II $5 \alpha$-RIs such as finasteride are commonly used to stabilize hair loss and promote regrowth.

Although BPH itself is an independent risk factor for erectile dysfunction (ED), evidence suggests that $5 \alpha$-RIs such as finasteride may independently increase the risk of adverse sexual side effects potentially through inducing androgen deficiency by inhibiting $5 \alpha-R$ and reducing available $5 \alpha$-DHT [7,8]. Actually, patients with $\mathrm{BPH}$ who used finasteride to treat their disease experienced worsening erectile function (EF) that did not resolve with continued treatment, although some studies reported that continuous treatment with finasteride decreased ED [8]. These sexual side effects are persistent or irreversible in some patients. The importance of $5 \alpha$-DHT in development and maintenance of male sexual organs and its function have been noted in several studies [9,10]. However, many investigators believe that side effects of $5 \alpha$-RIs will disappear with continuous treatment. Furthermore, side effects of $5 \alpha-R I s$ on $\mathrm{EF}$ have received minimal attention and deemed clinically less important $[7,8,11,12]$. Considerable controversy exists regarding the severity and persistence of side effects of finasteride. Therefore, the aim of this review was to summarize current research studies on finasteride associated with ED.

\section{PATHOPHYSIOLOGY OF FINASTERIDE AND ERECTILE DYSFUNCTION}

Androgen plays an essential role in maintaining the structure and function of the penis. However, finasteride is an competitive inhibitor of type II $5 \alpha-\mathrm{R}$ [13]. Finasteride is developed to decrease the conversion of testosterone to its more potent metabolite $5 \alpha-\mathrm{DHT}$. Based on literatures, finasteride can achieve 70-80\% reduction in DHT [13]. Traish et al [14] have found that castration can decrease smooth muscle components and increase collagen tissue in the corpus cavernosum. Zhang et al [15] have reported that prolonged administration of $5 \alpha$-RIs in aged rats can attenuate erectile response to electrical stimulation of the cavernous nerve. Mechanisms responsible for such effects could be due to structural changes, decreased autophagy, aggravated ultrastructural injury of mitochondria, and increased apoptosis, of the smooth muscle cells of the $5 \alpha$-RIstreatment rat group [15].

Some clinical studies already showed that the $5 \alpha-R I s$ treatment caused the increase of serum testosterone levels. Roehrborn et al [16] reported that finasteride treatment led to a significant increase relative to placebo in serum testosterone. Also, Hong et al [17] reported that dutasteride treatment in men with $\mathrm{BPH}$ led to a significant increase in serum testosterone levels. Despite the increase of total testosterone after finasteride treatment, ED is more influenced by the decrease of DHT. Because, DHT has higher affinity for the androgen receptors and more effective at increasing nitric oxide (NO) synthase expression than total testosterone $[18,19]$.

$\mathrm{EF}$ is perceived in the brain where receptors for testosterones are widely distributed. $5 \alpha-\mathrm{R}$ activity is also found in neurons, oligodendrocytes, and astrocytes [20]. Neuronal and glial cell development may be promoted by androgens metabolized by $5 \alpha-R$ [21]. Finasteride can cross the blood-brain barrier and inhibit the production of DHT throughout the central nervous system [22]. It is biologically plausible that a lack of DHT or another $5 \alpha$-reduced hormone is responsible for a decrease in libido resulting in ED [23]. Because pathogenesis of ED is multifactorial and involve psychogenic and organic components [24], decrease of the libido caused by finasteride can contribute to the development of ED.

We investigated the ability of reactive oxygen species 
(ROS) to evoke endoplasmic reticulum (ER) stress in a finasteride animal model and found that the unfolded protein response (UPR) effectors (glucose-regulated protein-78, phosphorylated c-jun-N-terminal kinase and phosphorylated inositol-requiring transmembrane kinase/endoribonuclease) and apoptotic markers (procaspase-3 and cleaved caspase-3) were triggered in a ROSdependent manner (Fig. 1) [25]. The overproduction of ROS induce a decrease in the bioavailability of NO in the corpus cavernosum, impairing vascular smooth muscle relaxation through the NO-cyclic guanosine monophosphate pathway [26,27]. ROS interfere with the folding process of polypeptides in the ER, therefore ER stress appears to damage vascular endothelial cells and compromise vascular relaxation of the corpus cavernosum [28]. In our opinion, ER stress caused by finasteride could be a mechanism of leading to ED. In addition to basic research, a large and randomized prospective clinical studies should be required to clarify these findings.

\section{RECENT RESEARCH OF FINASTERIDE FOR BENIGN PROSTATIC HYPERPLASIA AND ERECTILE DYSFUNCTION}

Most studies in this review have revealed that finasteride for BPH is correlated with ED. A summary of findings discussed below is presented in Table 1.

Recently, Corona et al [29] have conducted a comprehensive review and meta-analysis for ED in subjects treated with $5 \alpha-\mathrm{RIs}$, including finasteride for BPH. An extensive Medline Embase and Cochrane search was performed including the following words: 'finasteride', 'dutasteride', and 'benign prostatic hyperplasia'. Only placebo-controlled randomized clinical trials evaluating the effect of $5 \alpha-\mathrm{RIs}$ in subjects with BPH were considered. Of 383 retrieved articles, 17 were included in this study. Randomized clinical trials enrolled 24,463 in the active arm and 22,270 patients in the placebo arm, with a mean follow-up of 99 weeks and a mean age of 64.0 years. Overall, $5 \alpha-R I s$ increased the risk of hypoactive sexual desire (odds ratio $[\mathrm{OR}]=1.54 ; 95 \%$ confidence

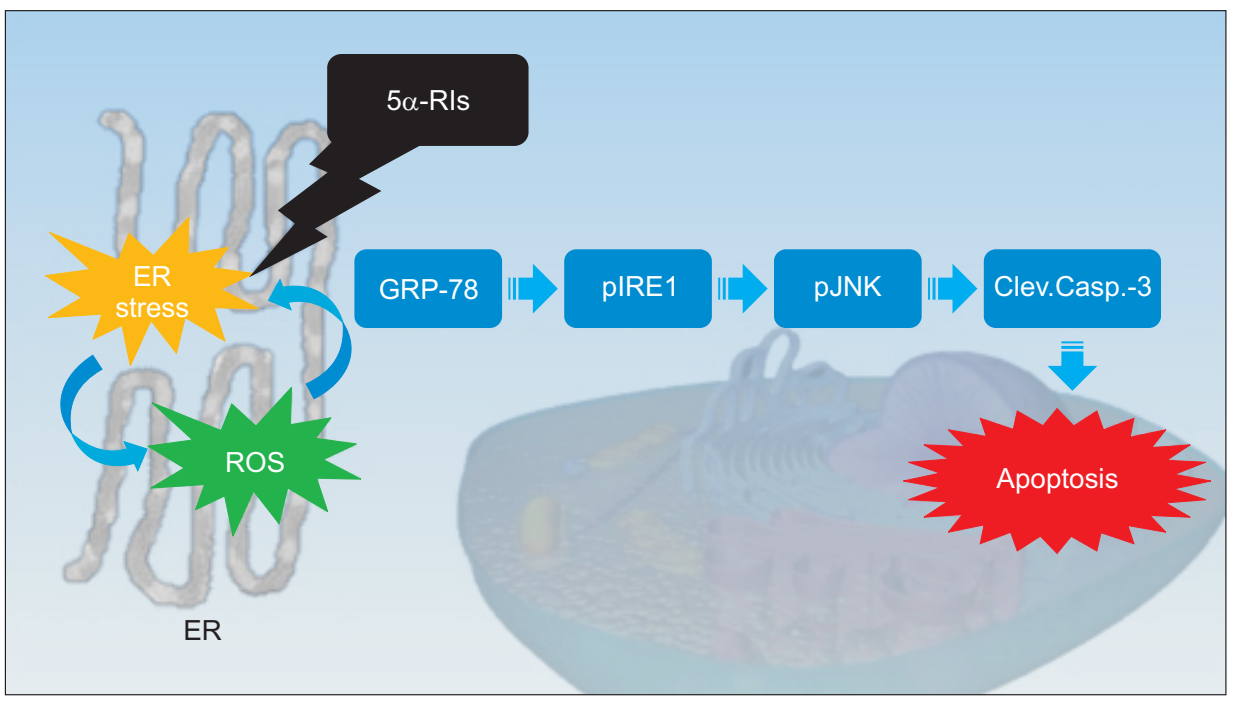

Fig. 1. Correlation between finasterideinduced oxidative stess and endoplasmic reticulum stress. ER: endoplasmic reticulum, $5 \alpha$-RIs: 5 -alpha reductase inhibitors, ROS: reactive oxygen species, GRP78: glucose-regulated protein-78, pIRE1: phosphorylated inositol-requiring transmembrane kinase/endoribonuclease, pJNK: phosphorylated c-jun- $\mathrm{N}$-terminal kinase, Clev.Casp.-3: cleaved caspase-3.

Table 1. Recent clinical studies of finasteride for BPH and ED

\begin{tabular}{llcll}
\multicolumn{1}{c}{ Study } & \multicolumn{1}{c}{ Study type } & Population & ED scale & Findings \\
\hline Corona et al (2017) [29] & Meta-analysis & 46,733 & Questionnaire & Increases the risk of ED \\
Liu et al (2016) [30] & Meta-analysis & 6,779 & Questionnaire & Increases the risk of ED \\
Hagberg et al (2016) [31] & Cohort & 71,849 & Medical record & No connections with ED \\
Traish et al (2015) [32] & Retrospective & 470 & Questionnaire & Increases the risk of ED \\
Fwu et al (2014) [33] & Multicenter, randomized & 2,783 & Questionnaire & Increases the risk of ED \\
\hline
\end{tabular}

BPH: benign prostatic hyperplasia, ED: erectile dysfunction. 
interval [C]], 1.29-1.82; $\mathrm{p}<0.0001)$ and $\mathrm{ED}(\mathrm{OR}=1.47 ; 95 \%$ CI, 1.29-1.68; $p<0.0001)$. Their data showed that the use of $5 \alpha-R I s$ significantly increased the risk of ED, ejaculatory dysfunction, and hypoactive sexual desire in subjects with $\mathrm{BPH}$.

In 2016, Liu et al [30] reported a meta-analysis and systematic review of randomized controlled trials for $\mathrm{ED}$ in subjects treated with $5 \alpha-\mathrm{RIs}$, including finasteride for BPH. Their study included 10 trials $(6,779$ patients) on the efficacy and safety of finasteride $5 \mathrm{mg}$ for BPH. The mean age of participants was 60.10 years across all studies. Pooled relative risk for sexual dysfunction was 2.56 (95\% confidence interval $[\mathrm{CT}]=1.48$ 4.42) and that for ED was 1.55 (95\% CI=1.14-2.12) in men with BPH. Sexual function also worsened with time in the $5 \alpha-\mathrm{RIs}$ groups. These findings suggested that $5 \alpha$-RIs were associated with $156 \%$ increased risk of sexual dysfunction for men with BPH. Furthermore, therapy lasting at least 1 year was associated with a statistically significant increased risk of adverse sexual effects compared with placebo $(\mathrm{p}=0.047$ and $\mathrm{p}=0.002$, respectively). Their study, the largest meta-analysis to date on the effect of $5 \alpha$-RIs on sexual function, demonstrated that $5 \alpha-$ RIs are associated with a significantly higher risk of ED than placebo in men with $\mathrm{BPH}$.

However, other studies have presented different results. Results of a study published in 2016 have provided evidence that $5 \alpha-R I s$ do not increase the risk of clinically meaningful incident ED in men who are free of sexual dysfunction, regardless of indication for use for BPH [31]. In the population with BPH (aged 40 years or more) $(n=71,849)$, the risk of $E D$ was not increased with use of $5 \alpha$-RIs only (incidence rate ratio: 0.92, 95\% CI: 0.85-0.99; OR: 0.94, 95\% CI: 0.85-1.03) or $5 \alpha-\mathrm{RIs}+\alpha$ blocker (incidence rate ratio: $1.09,95 \% \mathrm{CI}$ : 0.99-1.21; OR: 0.92, 95\% CI: 0.80-1.06) compared to the use of $\alpha$ blockers only. The risk remained null regardless of the number of prescriptions or timing of use. There were no proven causal connections between 5aRIs and symptoms of sexual dysfunction reported by patients.

In 2015, Traish et al [32] reported that long-term finasteride therapy results in worsening of $\mathrm{EF}$ and lower total testosterone levels in men with BPH. In their retrospective registry study, a cohort of 470 men aged between 47 and 68 years (mean: 57.78 44.81 years) were treated with finasteride $5 \mathrm{mg}$. A second cohort of 230 men aged between 52 and 72 years (mean: $62.62 \pm 4.65$ years) were treated with tamsulosin $0.4 \mathrm{mg}$. All men were followed-up for 45 months. At intervals of 3 months, plasma testosterone levels and International Index of Erectile Function (IIEF)-EF questionnaire scores were determined at each visit. Long-term treatment with finasteride therapy was associated with worsening of ED as shown by significant decrease in IIEF-EF scores in men treated with finasteride. The increase in ED due to finasteride was not resolved by continuing the treatment with finasteride. Most importantly, long-term finasteride therapy resulted in reduced total testosterone levels that contributed to a state of hypogonadism [32].

In MTOPS (Medical Therapy of Prostatic Symptoms) study, a multicenter, randomized, double-blind, placebo controlled clinical trial was performed with time to $\mathrm{BPH}$ progression as the primary outcome and change in sexual function as the secondary outcome [33]. They analyzed records of 2,783 men who completed the inventory at baseline and at least once during follow-up [33]. These men had an average age of $62.6 \pm 7.3$ years. Treatment with finasteride or finasteride combined therapy with doxazosin was associated with worsening sexual function compared to placebo. There was no significant difference in changes in sexual function in men assigned to doxazosin alone compared to placebo.

In 2012, Zhang et al [34] reported that finasteride treatment for 4 weeks reduced the weight of the corpus cavernosum without appearing to affect erectile responses to electrical stimulation of the cavernous nerve in a rat model. Adult male Sprague-Dawley rats were divided into four groups (n=25/group): (i) control; (ii) castration; (iii) castration with testosterone replacement; and (iv) oral finasteride treatment. Four weeks later, erectile function was measured by the ratio of intracavernosal pressure and mean arterial blood pressure upon electrical stimulation of the cavernous nerve. Tissue weights of the corpus cavernosum and prostate were reduced by $25.9 \%$ and $92.3 \%$ in group 4 compared to those in group 1 (both $\mathrm{p}<0.001$ ). However, there was no significant difference in serum testosterone concentration or erectile function between groups 4 and 1 . The author's explain that as erection is a complex process involving important signaling in the brain, further studies are necessary to demonstrate the long-term effects of finasteride on both central and peripheral neural pathways of erection. However, nobody knows that the erectile response to electrical field stimulation may 
Table 2. Recent clinical studies of finasteride for MAA and ED

\begin{tabular}{llcll}
\multicolumn{1}{c}{ Study } & \multicolumn{1}{c}{ Study type } & Population & ED scale & Findings \\
\hline Liu et al (2016) [30] & Meta-analysis & 4,493 & Questionnaire & No connections with ED \\
Hagberg et al (2016) [31] & Cohort & 12,346 & Medical record & No connections with ED \\
Chiriacò et al (2016) [35] & Retrospective & 79 & Questionnaire & Increases the risk of ED \\
Narasimhalu (2015) [36] & Randomized & 586 & Questionnaire & No connections with ED \\
\hline
\end{tabular}

MAA: male androgenic alopecia, ED: erectile dysfunction.

be different comparing with normal physiologic EF in threshold to stimulation.

\section{RECENT RESEARCH OF FINASTERIDE FOR MALE ANDROGENETIC ALOPECIA AND ERECTILE DYSFUNCTION}

Most studies included in this review revealed that finasteride for MAA was not correlated with ED. A summary of findings discussed below is presented in Table 2.

Recently, Liu et al [30] reported a meta-analysis and a systematic review of randomized controlled trials for $\mathrm{ED}$ in subjects treated with $5 \alpha-\mathrm{RI}$ s, including finasteride for MAA. Their study included three trials $(4,493$ patients) on the efficacy and safety of finasteride and dutasteride for MAA. The mean age of participants was 60.10 years across all studies. Pooled relative risk for sexual dysfunction was 1.21 (95\% CI: 0.85-1.72) and that for ED was 0.66 (95\% CI: 0.20-2.25) in men with MAA. Also, there was no significant association between duration of therapy and adverse sexual effects in men with MAA. Evidence from these randomized controlled trials suggested that the association between finasteride and ED was not statistically significant in men with MAA.

Hagberg et al [31] have conducted a cohort study using Clinical Practice Research Datalink for ED in subjects treated with $5 \alpha-R I s$, including finasteride for MAA. For the MAA population (aged 18-59 years) $(n=12,346)$, the risk of ED was not increased for users of finasteride $1 \mathrm{mg}$ compared to unexposed men with MAA (incidence rate ratio: 1.03, 95\% CI: 0.73-1.44; OR: 0.95, 95\% CI: 0.64-1.41).

However, other studies have revealed different results. In 2016, Chiriacò et al [35] showed that finasteride therapy for MAA resulted in worsening of ED. In their retrospective registry study, among 79 participants, 34\% were white Italians with mean age of $33.4 \pm 7.60$ years, mean duration of finasteride use of $27.3 \pm 33.21$ months, and mean time from finasteride discontinuation of $44.1 \pm 34.20$ months. Symptoms were investigated by an ad hoc 100 questions' questionnaire and validated Arizona Sexual Experience Scale and Aging Male Symptom Scale questionnaires. By validated Arizona Sexual Experience Scale questionnaire, $40.5 \%$ of participants declared that getting and keeping erection were very difficult while $3.8 \%$ of participants never achieved erection. Reaching orgasm was declared to be very difficult by $16.5 \%$ of participants and never achieved was declared by $2.5 \%$ of participants. By the ad hoc questionnaire, the most frequent sexual symptoms referred were loss of penis sensitivity (87.3\%), decreased ejaculatory force (82.3\%), and low penile temperature (78.5\%). In their study, unexpectedly loss of penis sensitivity was more frequent than severe ED while loss of muscle tone/mass was affecting half of these subjects.

In 2015, Narasimhalu [36] reported results of randomized controlled trials for ED in subjects treated with finasteride for MAA. A questionnaire based on the IIEF was given to approximately 586 patients with MAA who were being treated with finasteride $1 \mathrm{mg}$ for an average of 16 weeks. Statistical analysis of results showed no significant difference in scores between patients taking finasteride and those in the control group. This study suggested that ED was not a side effect of finasteride. In addition, 16 weeks with finasteride $1 \mathrm{mg}$ did not have enough dosage to result in symptomatic ED because patients with MAA were too young in age to have ED.

\section{COMMENTS}

Although there is not enough evidence to prove the relationship between finasteride and ED, the nocebo effect should be taken into account when studying 
sexual effects of finasteride [37]. Most studies in this review found that finasteride for $\mathrm{BPH}$ was correlated with ED whereas finasteride for MAA was not correlated with ED.

In our opinion, such difference in outcome might be due to different doses of $\mathrm{BPH}$ and MAA treatment with finasteride. Related studies have reported a lower occurrence of sexual adverse events with lower dose of finasteride $[37,38]$. This suggests that different risks of $\mathrm{ED}$ between the two groups (finasteride for $\mathrm{BPH}$ and finasteride for MAA) might be related to dosage of finasteride.

And the most important factor is difference of age in BPH and MAA patients. BPH patients were much older than MAA patients who participated in these clinical studies. Several studies have reported that the prevalence of $\mathrm{ED}$ is $2 \%$ to $9 \%$ in the decades from 40 to 49 years and $20 \%$ to $40 \%$ for men aged $<69$ years. However, the prevalence in men in their 70s and 80s is much higher [39]. The ageing process is accompanied by a progressively increasing of organic impairment. Therefore, the more the global population ages, the more they are affected by several comorbidities [40-46]. In elderly patients, finasteride seems to have more adverse effects on sexual dysfunction such as ED.

Data from epidemiological studies suggest a link between symptoms of $\mathrm{BPH}$ and $\mathrm{ED}$ in older men regardless of age, co-morbidity, or lifestyle [47-50]. Possible connection between these two diseases lies in the mechanism of action of $\alpha-1$ adrenergic receptor. These receptors might increase the tone of smooth muscle cells of the prostatic capsule and bladder neck in these patients. Penile erection depends on a balanced contraction and relaxation of the cavernous smooth muscle [51]. In ED, the aforementioned receptors affect noradrenaline and androgens and favor the contraction of smooth muscle. This may complicate the process of relaxation of the same muscle and lead to ED [52]. In $\mathrm{BPH}$ compared to MAA, the correlation between $\mathrm{BPH}$ and ED seems to have more influence than the adverse effect of finasteride on ED.

Although most studies in this review found that finasteride for MAA was not correlated with ED, some patients reported side effects of finasteride associated with sexual dysfunction, including ED, male infertility, and loss of libido [8,53-57]. Di Loreto et al [58] have suggested a mechanism of relationship between ED and finasteride in MAA patients. Androgen receptor and nerve density in foreskin prepuce specimens are associated with persistent sexual side effects, including loss of sensitivity in the genital area due to former finasteride use against MAA. In their study, 8 males (aged 29-43 years) reported ED, including loss of penis sensitivity over 6 months after discontinuation of finasteride. Controls were 11 otherwise healthy matched men (aged 23-49 years) who had undergone circumcision for phimosis. They never took finasteride or analogues. Differences in androgen receptor expression and nerve density in different portions of dermal prepuce were evaluated in these two groups. Density of nuclear androgen receptor in stromal and epithelial cells was found to be higher in cases (mean $40.0 \%$, and $80.6 \%$ of positive cells, respectively) than that in controls (mean $23.4 \%$, and $65.0 \%$ of positive cells, respectively) ( $\mathrm{p}=0.023$ and $\mathrm{p}=0.043$, respectively). This provides the first evidence of molecular and objective difference between patients with long-term adverse sexual effects after finasteride use vs. drug untreated healthy controls in certain tissues.

ED induced by finasteride might be due to ER stress. In the present study, Finasteride resulted in reduced fertility and increased ER stress and apoptotic markers in the SD rats [25]. Sperm damage occurs when oxidative stress overcomes natural antioxidant defenses. Accumulation of free radicals coupled with an increase in oxidative stress has been implicated in the pathogenesis of several disease states. Oxidative stress is a crucial regulator of ER function and activation of the UPR in disease conditions, as ER stress and increased oxidative stress production occur concurrently [26-28]. Oxidative stress can lead to ED by endothelial dysfunction in part through the activation of ER stress. Therefore, the mechanism of ED associated with finasteride needs to be clarified in the future.

Finally, we have to worry about the unexpected occurrences of $5 \alpha-$ RIs inducing sexual problems during the treatment in young patients who didn't have sexual problems before the treatment [23]. In the aged BPH patients who had been treated with $5 \alpha-\mathrm{RIs}$, they frequently complained the decrease in ejaculation volume and of libido. Similarly, when a young patient who has MAA and is treated with the $5 \alpha-\mathrm{RIs}$ treatment, the patient could also complain the same side effects. Furthermore, it should be noted that patients might not be able to recover from the sexual problems even after the discontinuation of the $5 \alpha-\mathrm{RI}$, and as a result might 
significantly deteriorate their quality of life. Therefore, physicians should discuss with their patients the possible long-term effect of finasteride on sexual function, even in MAA patients.

\section{CONCLUSION}

We reviewed most recent studies of finasteride treatment for BPH and MAA and the occurrence of ED. Evidence from clinical studies suggested that finasteride was associated with increased adverse effects on ED in men with BPH. However, such association was not statistically significant in men with MAA. Some studies reported that finasteride had side effects on sexual dysfunction, including ED, male infertility, ejaculatory dysfunction, and loss of libido, even in MAA patients. Therefore, well-designed randomized controlled trials are needed to determine the mechanism and effects of finasteride on ED. Physicians needs to discuss the possible long-term effect of finasteride ED with their patients, especially in the young healthy patients.

\section{ACKNOWLEDGEMENTS}

This study was supported by grants from the Korean Healthcare Technology R\&D Project, Ministry for Health, Welfare, \& Family Affairs, Republic of Korea (H114C0018). The Korean Healthcare Technology R\&D Project, Ministry for Health, Welfare \& Family Affairs, Republic of Korea had no role in the design or conduct of the study; collection, management, analysis, or interpretation of the data; or preparation, review, or approval of the manuscript. The content of this article is solely the responsibility of the authors and does not necessarily represent the official views of the Korean Healthcare Technology R\&D Project, Ministry for Health, Welfare, \& Family Affairs, Republic of Korea. All researchers are receiving support from this grant, but there are no financial or other potential conflicts of interest to declare.

Jong Kwan Park is a consultant and speaker for DongA Pharmaceutical Company, Yongin, Kyounggi-do, Republic of Korea, and has received unconditional research grants from that company. However, the company had no role in the design or conduct of the study; collection, management, analysis, or interpretation of the data; or preparation, review, or approval of the manuscript.

\section{Disclosure}

The authors have no potential conflicts of interest to disclose.

\section{Author Contribution}

Research conception \& design: Shin YS, Park JK. Data acquisition: Shin YS, Karna KK, Choi BR, Park JK. Data analysis and interpretation: Shin YS, Park JK. Drafting of the manuscript: Shin YS, Park JK. Critical revision of the manuscript: Shin YS, Park JK. Approval of final manuscript: all authors.

\section{REFERENCES}

1. Shin YS, Park JK. Changes in surgical strategy for patients with benign prostatic hyperplasia: 12-year single-center experience. Korean J Urol 2011;52:189-93.

2. Shin YS, Zhang LT, Zhao C, Kim YG, Park JK. Twelve-week, prospective, open-label, randomized trial on the effects of an anticholinergic agent or antidiuretic agent as add-on therapy to an alpha-blocker for lower urinary tract symptoms. Clin Interv Aging 2014;9:1021-30.

3. Shin YS, Lee JW, Kim MK, Jeong YB, Park SC. Early dutasteride monotherapy in men with detectable serum prostatespecific antigen levels following radical prostatectomy: a prospective trial. Investig Clin Urol 2017;58:98-102.

4. Yamana K, Labrie F, Luu-The V. Human type $35 \alpha$-reductase is expressed in peripheral tissues at higher levels than types 1 and 2 and its activity is potently inhibited by finasteride and dutasteride. Horm Mol Biol Clin Investig 2010;2:293-9.

5. Severi G, Sinclair R, Hopper JL, English DR, McCredie MR, Boyle $\mathrm{P}$, et al. Androgenetic alopecia in men aged 40-69 years: prevalence and risk factors. Br J Dermatol 2003;149:1207-13.

6. Gan DC, Sinclair RD. Prevalence of male and female pattern hair loss in Maryborough. J Investig Dermatol Symp Proc 2005; 10:184-9.

7. Traish AM. $5 \alpha$-reductases in human physiology: an unfolding story. Endocr Pract 2012;18:965-75.

8. Traish AM, Hassani J, Guay AT, Zitzmann M, Hansen ML. Adverse side effects of $5 \alpha$-reductase inhibitors therapy: persistent diminished libido and erectile dysfunction and depression in a subset of patients. J Sex Med 2011;8:872-84.

9. Baldinotti F, Majore S, Fogli A, Marrocco G, Ghirri P, Vuerich $M$, et al. Molecular characterization of 6 unrelated Italian patients with 5alpha-reductase type 2 deficiency. J Androl 2008;29:20-8.

10. MacLaughlin DT, Donahoe PK. Sex determination and dif- 
ferentiation. N Engl J Med 2004;350:367-78.

11. Andriole GL, Bostwick DG, Brawley OW, Gomella LG, Marberger M, Montorsi F, et al. Effect of dutasteride on the risk of prostate cancer. N Engl J Med 2010;362:1192-202.

12. Thompson IM, Goodman PJ, Tangen CM, Lucia MS, Miller GJ, Ford LG, et al. The influence of finasteride on the development of prostate cancer. N Engl J Med 2003;349:215-24.

13. Amory JK, Wang C, Swerdloff RS, Anawalt BD, Matsumoto AM, Bremner WJ, et al. The effect of 5alpha-reductase inhibition with dutasteride and finasteride on semen parameters and serum hormones in healthy men. J Clin Endocrinol Metab 2007;92:1659-65.

14. Traish AM, Toselli P, Jeong SJ, Kim NN. Adipocyte accumulation in penile corpus cavernosum of the orchiectomized rabbit: a potential mechanism for veno-occlusive dysfunction in androgen deficiency. J Androl 2005;26:242-8.

15. Zhang MG, Wang XJ, Shen ZJ, Gao PJ. Long-term oral administration of $5 \alpha$-reductase inhibitor attenuates erectile function by inhibiting autophagy and promoting apoptosis of smooth muscle cells in corpus cavernosum of aged rats. Urology 2013;82:743.e9-15.

16. Roehrborn CG, Lee M, Meehan A, Waldstreicher J; PLESS Study Group. Effects of finasteride on serum testosterone and body mass index in men with benign prostatic hyperplasia. Urology 2003;62:894-9.

17. Hong SK, Min GE, Ha SB, Doo SH, Kang MY, Park HJ, et al. Effect of the dual 5alpha-reductase inhibitor, dutasteride, on serum testosterone and body mass index in men with benign prostatic hyperplasia. BJU Int 2010;105:970-4.

18. Gur S, Kadowitz PJ, Hellstrom WJ. Effects of 5-alpha reductase inhibitors on erectile function, sexual desire and ejaculation. Expert Opin Drug Saf 2013;12:81-90.

19. Traish AM. Negative impact of testosterone deficiency and $5 \alpha$-reductase inhibitors therapy on metabolic and sexual function in men. Adv Exp Med Biol 2017;1043:473-526.

20. Melcangi RC, Magnaghi V, Martini L. Steroid metabolism and effects in central and peripheral glial cells. J Neurobiol 1999;40:471-83.

21. Melcangi RC, Riva MA, Fumagalli F, Magnaghi V, Racagni G, Martini L. Effect of progesterone, testosterone and their 5 alpha-reduced metabolites on GFAP gene expression in type 1 astrocytes. Brain Res 1996;711:10-5.

22. Merck Co. Propecia prescribing information. Whitehouse Station (NJ): Merck Co.; 2013.

23. Irwig MS, Kolukula S. Persistent sexual side effects of finasteride for male pattern hair loss. J Sex Med 2011;8:1747-53.

24. Nguyen HMT, Gabrielson AT, Hellstrom WJG. Erectile dysfunction in young men-a review of the prevalence and risk factors. Sex Med Rev 2017;5:508-20.

25. Soni KK, Shin YS, Choi BR, Karna KK, Kim HK, Lee SW, et al. Protective effect of DA-9401 in finasteride-induced apoptosis in rat testis: inositol requiring kinase 1 and c-Jun $\mathrm{N}$ terminal kinase pathway. Drug Des Devel Ther 2017;11:296979.

26. Nishikawa T, Edelstein D, Du XL, Yamagishi S, Matsumura T, Kaneda Y, et al. Normalizing mitochondrial superoxide production blocks three pathways of hyperglycaemic damage. Nature 2000;404:787-90.

27. Bivalacqua TJ, Kendirci M, Champion HC, Hellstrom WJ, Andersson KE, Hedlund P. Dysregulation of cGMP-dependent protein kinase 1 (PKG-1) impairs erectile function in diabetic rats: influence of in vivo gene therapy of PKG1alpha. BJU Int 2007;99:1488-94.

28. Cheng YS, Cong XD, Dai DZ, Zhang Y, Dai Y. Argirein alleviates corpus cavernosum dysfunction by suppressing proinflammatory factors p66Shc and ER stress chaperone Bip in diabetic rats. J Pharm Pharmacol 2013;65:94-101.

29. Corona G, Tirabassi G, Santi D, Maseroli E, Gacci M, Dicuio $M$, et al. Sexual dysfunction in subjects treated with inhibitors of $5 \alpha$-reductase for benign prostatic hyperplasia: a comprehensive review and meta-analysis. Andrology 2017;5:671-8.

30. Liu L, Zhao S, Li F, Li E, Kang R, Luo L, et al. Effect of $5 \alpha$-reductase inhibitors on sexual function: a meta-analysis and systematic review of randomized controlled trials. J Sex Med 2016;13:1297-310.

31. Hagberg KW, Divan HA, Persson R, Nickel JC, Jick SS. Risk of erectile dysfunction associated with use of 5- $\alpha$ reductase inhibitors for benign prostatic hyperplasia or alopecia: population based studies using the Clinical Practice Research Datalink. BMJ 2016;354:i4823.

32. Traish AM, Haider KS, Doros G, Haider A. Finasteride, not tamsulosin, increases severity of erectile dysfunction and decreases testosterone levels in men with benign prostatic hyperplasia. Horm Mol Biol Clin Investig 2015;23:85-96.

33. Fwu CW, Eggers PW, Kirkali Z, McVary KT, Burrows PK, Kusek JW. Change in sexual function in men with lower urinary tract symptoms/benign prostatic hyperplasia associated with long-term treatment with doxazosin, finasteride and combined therapy. J Urol 2014;191:1828-34.

34. Zhang MG, Wu W, Zhang CM, Wang XJ, Gao PJ, Lu YL, et al. Effects of oral finasteride on erectile function in a rat model. J Sex Med 2012;9:1328-36.

35. Chiriacò G, Cauci S, Mazzon G, Trombetta C. An observational retrospective evaluation of 79 young men with longterm adverse effects after use of finasteride against androgenetic alopecia. Andrology 2016;4:245-50. 
36. Narasimhalu CR. Randomized questionnaire based case-control research study on evaluation of sexual function in indian patients taking oral finasteride for androgenetic alopecia. Dermatol Ther (Heidelb) 2015;5:231-4.

37. Mondaini N, Gontero P, Giubilei G, Lombardi G, Cai T, Gavazzi $\mathrm{A}$, et al. Finasteride $5 \mathrm{mg}$ and sexual side effects: how many of these are related to a nocebo phenomenon? J Sex Med 2007;4:1708-12.

38. Olsen EA, Hordinsky M, Whiting D, Stough D, Hobbs S, Ellis $\mathrm{ML}$, et al. The importance of dual 5alpha-reductase inhibition in the treatment of male pattern hair loss: results of a randomized placebo-controlled study of dutasteride versus finasteride. J Am Acad Dermatol 2006;55:1014-23.

39. De Almeida Claro J, Kaufmann OG, Alarcon G, Aguiar W, Nadozza A Jr, Ortiz V, et al. Could a rural lifestyle decrease the prevalence of erectile dysfunction? BJU Int 2007;99:127-9.

40. Montorsi F, Adaikan G, Becher E, Giuliano F, Khoury S, Lue TF, et al. Summary of the recommendations on sexual dysfunctions in men. J Sex Med 2010;7:3572-88.

41. DeLay KJ, Haney N, Hellstrom WJ. Modifying risk factors in the management of erectile dysfunction: a review. World J Mens Health 2016;34:89-100.

42. NIH Consensus Conference. Impotence. NIH Consensus Development Panel on Impotence. JAMA 1993;270:83-90.

43. Lewis RW, Fugl-Meyer KS, Corona G, Hayes RD, Laumann EO, Moreira ED Jr, et al. Definitions/epidemiology/risk factors for sexual dysfunction. J Sex Med 2010;7:1598-607.

44. Salonia A, Capogrosso P, Clementi MC, Castagna G, Damiano $\mathrm{R}$, Montorsi F. Is erectile dysfunction a reliable indicator of general health status in men? Arab J Urol 2013;11:203-11.

45. Khanzada U, Khan SA, Hussain M, Adel H, Masood K, Adil SO, et al. Evaluation of the causes of erectile dysfunction in patients undergoing penile doppler ultrasonography in Pakistan. World J Mens Health 2017;35:22-7.

46. Corona G, Lee DM, Forti G, O’Connor DB, Maggi M, O’Neill TW, et al. Age-related changes in general and sexual health in middle-aged and older men: results from the European Male Ageing Study (EMAS). J Sex Med 2010;7:1362-80.

47. Adegun PT, Areo PO, Solomon A, Dada SA, Adebayo PB. Erectile dysfunction in men with and without lower urinary tract symptoms in Nigeria. World J Mens Health 2017;35:107-
14.

48. Seftel AD, de la Rosette J, Birt J, Porter V, Zarotsky V, Viktrup L. Coexisting lower urinary tract symptoms and erectile dysfunction: a systematic review of epidemiological data. Int J Clin Pract 2013;67:32-45.

49. Won JE, Chu JY, Choi HC, Chen Y, Park HJ, Dueñas HJ. Safety and effectiveness of once-daily tadalafil $(5 \mathrm{mg})$ therapy in Korean men with benign prostatic hyperplasia/lower urinary tract symptoms in a real-world clinical setting: results from a post-marketing surveillance study. World J Mens Health 2018;36:161-70.

50. Kardasevic A, Milicevic S. The correlation between prostate volume in patients with benign prostatic hyperplasia in relation to erectile dysfunction. Med Arch 2016;70:449-52.

51. Choi BR, Kim HK, Park JK. Penile erection induced by scoparone from artemisia capillaris through the nitric oxide-cyclic guanosine monophosphate signaling pathway. World J Mens Health 2017;35:196-204.

52. O'Leary MP. LUTS, ED, QOL: alphabet soup or real concerns to aging men? Urology 2000;56(5 Suppl 1):7-11.

53. Singh MK, Avram M. Persistent sexual dysfunction and depression in finasteride users for male pattern hair loss: a serious concern or red herring? J Clin Aesthet Dermatol 2014;7:51-5.

54. Ganzer CA, Jacobs AR, Iqbal F. Persistent sexual, emotional, and cognitive impairment post-finasteride: a survey of men reporting symptoms. Am J Mens Health 2015;9:222-8.

55. Irwig MS. Persistent sexual and nonsexual adverse effects of finasteride in younger men. Sex Med Rev 2014;2:24-35.

56. Irwig MS. Decreased alcohol consumption among former male users of finasteride with persistent sexual side effects: a preliminary report. Alcohol Clin Exp Res 2013;37:1823-6.

57. Traish AM, Mulgaonkar A, Giordano N. The dark side of $5 \alpha$-reductase inhibitors' therapy: sexual dysfunction, high Gleason grade prostate cancer and depression. Korean J Urol 2014;55:367-79.

58. Di Loreto C, La Marra F, Mazzon G, Belgrano E, Trombetta C, Cauci S. Immunohistochemical evaluation of androgen receptor and nerve structure density in human prepuce from patients with persistent sexual side effects after finasteride use for androgenetic alopecia. PLoS One 2014;9:e100237. 